\title{
Adaptive State Tracking Control with Actuator Nonlinearities and Failures
}

\author{
Liyan Wen ${ }^{1,2}$, Gang Tao ${ }^{2}$ \\ ${ }^{1}$ College of Automation Engineering \\ Nanjing University of Aeronautics and Astronautics, Nanjing, 210016, China \\ wenliyan8710@163.com \\ ${ }^{2}$ Department of Electrical and Computer Engineering \\ University of Virginia, Charlottesville, VA 22903 \\ gt9s@ virginia.edu
}

\begin{abstract}
In this paper, adaptive control is developed for an $M$-input linear time-invariant system with actuator failures and nonlinearities in presence of an unknown triangular matrix $\Lambda$. Three technical problems are solved: (i) the development of a simple adaptive controller without the use of an additional compensator system, which ensures desired stability and state tracking properties; (ii) the extension of the developed adaptive control scheme the case when $\Lambda$ is an unknown triangular matrix; and (iii) the development of an adaptive control scheme for the case when some of the actuators fail during the system operation, that is, some components of $u(t)=\left[u_{1}, u_{2}, \ldots, u_{M}\right]^{T}$ become uncontrollable by the feedback control signals.
\end{abstract}

Keywords: Adaptive control, state tracking, nonlinearity, actuator failures.

\section{Introduction}

Adaptive control has been studied for dealing with system uncertainties from the plant and external disturbances to ensure desired tracking performance [1, 2, 3, 4, 5, 6]. In this paper, we consider the adaptive control problems for an $M$-input linear time-invariant system:

$$
\dot{x}(t)=A x(t)+B \Lambda u(t)+B \Theta^{*} \phi(x), x(t) \in R^{n}, u(t) \in R^{M},
$$

where $(A, B)$ is a known and controllable pair, $\Lambda \in R^{M \times M}$ is an unknown matrix, $\Theta^{*} \in R^{M \times s}$ is an unknown constant matrix, and $\phi(x) \in R^{s}$ is a known vector. Systems with such a structure have recently attracted considerable attentions in the literature [7, 8, 9], mainly for $\Lambda=\operatorname{diag}\left\{\lambda_{1}, \lambda_{2}, \ldots, \lambda_{M}\right\}$ is an unknown and diagonal matrix with $\lambda_{i} \neq 0$ for $i=1,2, \ldots, M$. Typical current solutions to adaptive control of such systems are based on the introduction of another $n$-dimensional compensator systems and the design of an adaptive controller for the augmented system, for achieving some state tracking.

Different from the existing results in [7, 8, 9], in this paper, we will solve three technical problems: (i) the development of a simple adaptive controller without the use of an additional compensator system, which ensures desired stability and state tracking properties; (ii) the extension of the developed adaptive control scheme to the case when $\Lambda$ is an unknown triangular matrix; and (iii) the development of an adaptive control scheme for the case when some of the actuators fail during the system operation, that is, some components of $u(t)=\left[u_{1}, u_{2}, \ldots, u_{M}\right]^{T}$ become uncontrollable by the feedback control signals. 
The rest of this paper is organized as follows. In Section 2, adaptive control is studied for the plant with diagonal $\Lambda$. In Section 3 , adaptive control is studied for the triangular $\Lambda$ case. In Section 5 , simulation study is shown and conclusions are given in Section 6 .

\section{Adaptive Control Design for Diagonal $\Lambda$}

Consider an $M$-input linear time-invariant plant

$$
\dot{x}(t)=A x(t)+B \Lambda u(t)+B \Theta^{*} \phi(x),
$$

where $(A, B)$ is a known and controllable pair, $\Lambda=\operatorname{diag}\left\{\lambda_{1}, \lambda_{2}, \ldots, \lambda_{M}\right\}$ is an unknown and diagonal matrix with $\lambda_{i} \neq 0$ for $i=1,2, \ldots, M, \Theta^{*} \in R^{M \times s}$ is an unknown matrix, and $\phi(x) \in R^{s}$ is a known vector. $x(t) \in R^{n}$ is the system state vector and $u(t) \in R^{M}$ is the control input vector. With $(A, B)$ known and controllable, we can find known constant matrices $K_{10} \in R^{n \times M}$ and $K_{20} \in R^{M \times M}$ such that

$$
A_{m}=A+B K_{10}^{T}, B_{m}=B K_{20}
$$

are known with $A_{m}$ being stable, for constructing a good reference model system

$$
\dot{x}_{m}(t)=A_{m} x_{m}(t)+B_{m} r(t), x_{m}(t) \in R^{n}, r(t) \in R^{M},
$$

where $r(t)$ is a bounded and piecewise continuous reference input.

We first introduce the parameter matrices

$$
K_{1}^{* T}=\Lambda^{-1} K_{10}^{T}, K_{2}^{*}=\Lambda^{-1} K_{20}, K_{3}^{*}=-\Lambda^{-1} \Theta^{*} .
$$

Then, for $K_{1}^{T}(t), K_{2}(t)$ and $K_{3}(t)$ being the estimates of $K_{1}^{* T} \in R^{M \times n}, K_{2}^{*} \in R^{M \times M}$ and $K_{3}^{*} \in R^{M \times s}$, we use the adaptive controller

$$
u(t)=K_{1}^{T}(t) x(t)+K_{2}(t) r(t)+K_{3}(t) \phi(x) .
$$

From the definitions of $K_{1}^{*}, K_{2}^{*}$ and $K_{3}^{*}$, we have

$$
B \Lambda\left(K_{1}^{* T} x(t)+K_{2}^{*} r(t)+K_{3}^{*} \phi(x)\right)=B\left(K_{10}^{T} x(t)+K_{20} r(t)-\Theta^{*} \phi(x)\right) .
$$

Introducing the parameter errors $\tilde{K}_{1}=K_{1}-K_{1}^{*}, \tilde{K}_{2}=K_{2}-K_{2}^{*}, \tilde{K}_{3}=K_{3}-K_{3}^{*}$, we express the control signal from 2.6) as

$$
u(t)=\tilde{K}_{1}^{T}(t) x(t)+\tilde{K}_{2}(t) r(t)+\tilde{K}_{3}(t) \phi(x)+K_{1}^{* T} x(t)+K_{2}^{*} r(t)+K_{3}^{*} \phi(x),
$$

and, in view of (2.3) and (2.7), the closed-loop system as

$$
\begin{aligned}
\dot{x}(t)= & A x(t)+B \Lambda\left(\tilde{K}_{1}^{T}(t) x(t)+\tilde{K}_{2}(t) r(t)+\tilde{K}_{3}(t) \phi(x)\right) \\
& +B \Lambda\left(K_{1}^{* T} x(t)+K_{2}^{*} r(t)+K_{3}^{*} \phi(x)\right)+B \Theta^{*} \phi(x) \\
= & A_{m} x(t)+B_{m} r(t)+B \Lambda\left(\tilde{K}_{1}^{T}(t) x(t)+\tilde{K}_{2}(t) r(t)+\tilde{K}_{3}(t) \phi(x)\right) .
\end{aligned}
$$

Then, in view of this equation and (2.4), the tracking error $e(t)=x(t)-x_{m}(t)$ satisfies

$$
\dot{e}(t)=A_{m} e(t)+B \Lambda\left(\tilde{K}_{1}^{T}(t) x(t)+\tilde{K}_{2}(t) r(t)+\tilde{K}_{3}(t) \phi(x)\right) .
$$


Introducing $\theta_{i}^{*}$ such that $\theta_{i}^{* T}$ is the $i$ th row of $\left[K_{1}^{* T}, K_{2}^{*}, K_{3}^{*}\right], i=1, \ldots, M$, letting $\theta_{i}$ be the estimate of $\theta_{i}^{*}$ and $\tilde{\theta}_{i}(t)=\theta_{i}(t)-\theta_{i}^{*}, i=1, \ldots, M$, and defining $\omega(t)=\left[x^{T}(t), r^{T}(t), \phi^{T}(x)\right]^{T} \in R^{n+M+s}$, we express 2.10) as

$$
\dot{e}(t)=A_{m} e(t)+B \Lambda\left[\begin{array}{c}
\tilde{\theta}_{1}^{T}(t) \omega(t) \\
\vdots \\
\tilde{\theta}_{M}^{T}(t) \omega(t)
\end{array}\right] .
$$

Letting $P=P^{T}>0$ satisfy $P A_{m}+A_{m}^{T} P=-Q$ for a chosen constant matrix $Q \in R^{n \times n}$ such that $Q=Q^{T}>0$, and $\bar{e}_{i}(t)$ be the $i$ th component of $e^{T}(t) P B, i=1, \ldots, M$, we design the adaptive law for $\theta_{i}(t)$ as

$$
\dot{\theta}_{i}(t)=-\operatorname{sign}\left[\lambda_{i}\right] \Gamma_{i} \bar{e}_{i}(t) \omega(t),
$$

where $\Gamma_{i}=\Gamma_{i}^{T}>0$ is a chosen constant adaptation gain matrix, and $\operatorname{sign}\left[\lambda_{i}\right]$ is the sign of $\lambda_{i}, i=1,2, \ldots, M$.

To analyze the adaptive control system performance, we consider the positive definite function $V\left(e, \tilde{\theta}_{i}, i=1,2, \ldots, M\right)=e^{T} P e+\sum_{i=1}^{M}\left|\lambda_{i}\right| \tilde{\theta}_{i}^{T} \Gamma_{i}^{-1} \tilde{\theta}_{i}$ and derive its time derivative along the trajectory of 2.11, and (2.12) as $\dot{V}=-e^{T}(t) Q e(t)$. From this result, the following Theorem 1 can be established next.

Theorem 1. The feedback controller (2.6) with the adaptive law (2.12), applied to the system (2.2), ensures that all signals in the closed-loop control system are bounded and the tracking error $e(t)=x(t)-x_{m}(t)$ satisfies: $\lim _{t \rightarrow \infty} e(t)=0$.

\section{Adaptive Control for Triangular $\Lambda$}

In this section, we solve the adaptive control problem for the case when $\Lambda$ in (1.1) is either an unknown lower triangular matrix or an unknown upper triangular matrix, using a simple controller structure derived from the above developed adaptive control scheme. When $\Lambda$ in 1.1 is an unknown upper triangular matrix, we use DU decomposition to decompose $\Lambda$ as $\Lambda=D^{*} U$, where $U$ is a unit upper triangular matrix, and

$$
D^{*}=\operatorname{diag}\left\{d_{1}^{*}, d_{2}^{*}, \cdots, d_{m}^{*}\right\}=\operatorname{diag}\left\{\Delta_{1}, \frac{\Delta_{2}}{\Delta_{1}}, \cdots, \frac{\Delta_{M}}{\Delta_{M-1}}\right\},
$$

with $\Delta_{i}, i=1,2, \cdots, M$, being the leading principal minors of $\Lambda$. For such an uncertain $\Lambda$, we make a basic assumption which is necessary for adaptive law designs.

Assumption 1. The leading principal minors of the unknown $\Lambda$ are non-zero and their signs are known.

With the adaptive controller 2.6, where $K_{1}, K_{2}$ and $K_{3}$ are the estimates of $K_{1}^{*}, K_{2}^{*}$ and $K_{3}^{*}$ :

$$
K_{1}^{* T}=\Lambda^{-1} K_{10}^{T}, K_{2}^{*}=\Lambda^{-1} K_{20}, K_{3}^{*}=-\Lambda^{-1} \Theta^{*},
$$

we express the closed-loop system as in the form:

$$
\dot{x}(t)=A_{m} x(t)+B_{m} r(t)+B \Lambda\left(u(t)-K_{1}^{* T} x(t)-K_{2}^{*} r(t)-K_{3}^{*} \phi(x)\right),
$$

in which $\Lambda$ is an unknown upper triangular matrix. Then, we have

$$
\begin{aligned}
\dot{x}(t) & =A_{m} x(t)+B_{m} r(t)+B D^{*} U\left(u(t)-K_{1}^{* T} x(t)-K_{2}^{*} r(t)-K_{3}^{*} \phi(x)\right) \\
& =A_{m} x(t)+B_{m} r(t)+B D^{*}\left(u(t)-(I-U) u(t)-U K_{1}^{* T} x(t)-U K_{2}^{*} r(t)-U K_{3}^{*} \phi(x)\right),
\end{aligned}
$$

CDSR 118-3 
where $I-U$ is an upper triangular matrix with zero diagonal elements. In view of this equation and (2.4), the tracking error $e(t)=x(t)-x_{m}(t)$ satisfies

$$
\dot{e}(t)=A_{m} e(t)+B D^{*}\left(u(t)-\Phi_{0}^{*} u(t)-\Phi_{1}^{* T} x(t)-\Phi_{2}^{*} r(t)-\Phi_{3}^{*} \phi(x)\right),
$$

where $\Phi_{0}^{*}=I-U \in R^{M \times M}, \Phi_{1}^{* T}=U K_{1}^{* T} \in R^{M \times n}, \Phi_{2}^{*}=U K_{2}^{*} \in R^{M \times M}$, and $\Phi_{3}^{*}=U K_{3}^{*} \in R^{M \times s}$. This error equation motivates the adaptive controller structure as

$$
u(t)=\Phi_{0} u(t)+\Phi_{1}^{T} x(t)+\Phi_{2} r(t)+\Phi_{3} \phi(x),
$$

where $\Phi_{0}, \Phi_{1}^{T}, \Phi_{2}$, and $\Phi_{3}$, are estimates of $\Phi_{0}^{*}, \Phi_{1}^{* T}, \Phi_{2}^{*}$ and $\Phi_{3}^{*}$, and the parameter matrix $\Phi_{0}$ has the same special upper triangular form as that of $\Phi_{0}^{*}$, that is,

$$
\Phi_{0}=\left[\begin{array}{ccccc}
0 & \phi_{12} & \phi_{13} & \cdots & \phi_{1 M} \\
0 & 0 & \phi_{13} & \cdots & \phi_{1 M} \\
\vdots & \vdots & \vdots & \vdots & \vdots \\
0 & 0 & \cdots & 0 & \phi_{M-1 M} \\
0 & 0 & \cdots & 0 & 0
\end{array}\right] \in R^{M \times M} .
$$

This special form ensures that

$$
\begin{aligned}
u_{M}(t) & =\left[\Phi_{1}^{T} x(t)+\Phi_{2} r(t)+\Phi_{3} \phi(x)\right]_{M}, \\
u_{M-1}(t) & =\phi_{M-1, M} u_{M}(t)+\left[\Phi_{1}^{T} x(t)+\Phi_{2} r(t)+\Phi_{3} \phi(x)\right]_{M-1}, \\
& \vdots \\
u_{2}(t) & =\sum_{i=3}^{M} \phi_{2 i} u_{i}(t)+\left[\Phi_{1}^{T} x(t)+\Phi_{2} r(t)+\Phi_{3} \phi(x)\right]_{2}, \\
u_{1}(t) & =\sum_{i=2}^{M} \phi_{2 i} u_{i}(t)+\left[\Phi_{1}^{T} x(t)+\Phi_{2} r(t)+\Phi_{3} \phi(x)\right]_{1},
\end{aligned}
$$

where $[v]_{i}$ is the $i$ th row of the vector $v$ and $\phi_{i j}$ is the component from the $i$ th row and the $j$ th column of $\Phi_{0}$.

With the special parameter structure 3.19 , we let $\Phi_{1 i}^{T}, \Phi_{2 i}$ and $\Phi_{3 i}$ be the $i$ th row of $\Phi_{1}^{T}(t), \Phi_{2}(t)$ and $\Phi_{3}(t)$, respectively, with $i=1,2, \cdots, M$, in (3.18). And define

$$
\begin{aligned}
\theta_{1} & =\left[\phi_{12}(t), \phi_{13}(t), \cdots, \phi_{1 M}(T), \Phi_{11}^{T}, \Phi_{21}, \Phi_{31}\right]^{T} \in R^{n+2 M+s-1}, \\
\theta_{2} & =\left[\phi_{23}(t), \cdots, \phi_{2 M}(T), \Phi_{12}^{T}, \Phi_{22}, \Phi_{32}\right]^{T} \in R^{n+2 M+s-2}, \\
& \vdots \\
\theta_{M-1} & =\left[\phi_{M-1, M}(t), \Phi_{1, M-1}^{T}, \Phi_{2, M-1}, \Phi_{3, M-1}\right]^{T} \in R^{n+M+s+1}, \\
\theta_{M} & =\left[\Phi_{1, M}^{T}, \Phi_{2, M}, \Phi_{3, M}\right]^{T} \in R^{n+M+s},
\end{aligned}
$$

as estimates of $\theta_{i}^{*}$ from the $i$ th rows of $\Phi_{0}^{*}, \Phi_{1}^{* T}, \Phi_{2}^{*}$, and $\Phi_{3}^{*}$. With 3.17, 3.18) and 3.23, we obtain

$$
\dot{e}(t)=A_{m} e(t)+B D^{*}\left[\begin{array}{c}
\tilde{\theta}_{1}^{T}(t) \omega_{1}(t) \\
\vdots \\
\tilde{\theta}_{M}^{T}(t) \omega_{M}(t)
\end{array}\right],
$$


where $\tilde{\theta}_{i}(t)=\theta_{i}-\theta_{i}^{*}, i=1,2, \cdots, M$, and

$$
\begin{aligned}
\omega_{1}(t) & =\left[u_{2}(t), u_{3}(t), \cdots, u_{M}(t), x^{T}, r^{T}(t), \phi^{T}(x)\right]^{T} \in R^{n+2 M+s-1}, \\
\omega_{2}(t) & =\left[u_{3}(t), u_{4}(t), \cdots, u_{M}(t), x^{T}, r^{T}(t), \phi^{T}(x)\right]^{T} \in R^{n+2 M+s-2}, \\
& \vdots \\
\omega_{M-1}(t) & =\left[u_{M}(t), x^{T}, r^{T}(t), \phi^{T}(x)\right]^{T} \in R^{n+M+s+1}, \\
\omega_{M}(t) & =\left[x^{T}, r^{T}(t), \phi^{T}(x)\right]^{T} \in R^{n+M+s} .
\end{aligned}
$$

Adaptive laws. Based on (3.24), the adaptive laws for the adaptive estimates $\theta_{i}(t)$ are chosen:

$$
\dot{\theta}_{i}(t)=-\operatorname{sign}\left[d_{i}^{*}\right] \Gamma_{i} \bar{e}_{i}(t) \omega_{i}(t), t \geq 0,
$$

where $\bar{e}_{i}(t)$ is the $i$ th component of $e^{T} P B$ with $P=P^{T}>0$ and $i=1, \cdots, M$, satisfying $P A_{m}+A_{m}^{T} P=-Q$, for a chosen constant matrix $Q \in R^{n \times n}$ such that $Q=Q^{T}>0, \Gamma_{i}=\Gamma_{i}^{T}>0$, and $\operatorname{sign}\left[d_{i}^{*}\right]$ is known (Assumption 1). To analyze the stability properties of the adaptive law (3.26), we consider the positive definite function

$$
V\left(e, \tilde{\theta}_{i}, i=1,2, \cdots, M\right)=e^{T} P e+\sum_{i=1}^{M}\left|d_{i}^{*}\right| \tilde{\theta}_{i}^{T} \Gamma_{i}^{-1} \tilde{\theta}_{i} .
$$

The time-derivative of $V\left(e, \tilde{\theta}_{i}\right)$, along the trajectory 3.26 , is

$$
\dot{V}=2 e^{T} P \dot{e}+2 \sum_{i=1}^{M}\left|d_{i}^{*}\right| \tilde{\theta}_{i}^{T} \Gamma_{i}^{-1} \dot{\theta}_{i}=-e^{T}(t) Q e(t) .
$$

Since $Q=Q^{T}>0$, 3.28 implies that the equilibrium state $e_{c}=0$, with $e_{c}(t)=\left[e^{T}, \tilde{\theta}_{1}^{T}, \cdots, \tilde{\theta}_{M}^{T}\right]^{T}$, of the closed-loop system consisting 3.24 and 3.26 is uniformly stable and its solution $e_{c}(t)$ is uniformly bounded. Therefore, $y(t), \theta_{i}(t), i=1,2, \cdots, M$, and $\dot{e}(t)$ are all bounded. And from (3.28), we obtain $e(t)=x(t)-x_{m}(t) \in L^{2}$, and so as $\lim _{t \rightarrow \infty} e(t)=0$. Then, we establish Theorem 2 .

Theorem 2. The feedback controller (3.18) with the adaptive control law (3.26), applied to the system (1.1) with an uncertain upper triangular matrix $\Lambda$, ensures that all signals in the closed-loop control system are bounded and the tracking error $e(t)$ satisfies: $\lim _{t \rightarrow \infty} e(t)=0$.

Remark 1. When $\Lambda$ is an unknown lower triangular matrix, LD decomposition can be adopted to decompose $\Lambda=L D^{*}$ with $L$ being a unit lower triangular matrix and $D$ defined as (3.13). Then, similar derivations as shown above can be made for designing an adaptive control input signal $u(t)$ in 3.18 (while, for this case, the signal component $u_{1}(t)$ will be first designed).

\section{Adaptive Control for Actuator Failures}

In this section, we solve the adaptive control problem when some actuators in the system fail at uncertain time instants, in uncertain failure patterns and with uncertain failure values. The type of actuator failures considered in this paper is characterized by some unknown inputs being stuck at some unknown fixed values that cannot be influenced by control action, which are modeled as

$$
u_{j}(t)=\bar{u}_{j}, t \geq t_{j}, j \in\{1,2, \ldots, M\},
$$


where the failure value $\bar{u}_{j}$, the failure time instant $t_{j}$ and the failure index $j$ are unknown. A typical example is an aircraft control surface (such as rudder, elevator, or stabilizer) stuck at some unknown fixed value not influenced by control. In the presence of failures, $u(t)$ can be expressed as

$$
u(t)=v(t)+\sigma(\bar{u}-v(t)),
$$

where $v(t)$ is the applied control input to be designed, and $\sigma=\operatorname{diag}\left\{\sigma_{1}, \sigma_{2}, \ldots, \sigma_{M}\right\}$ is the failure pattern matrix with $\sigma_{j}=1$ representing the $j$ th actuator fails, i.e., $u_{j}=\bar{u}_{j}$ and otherwise, $\sigma_{j}=0$, and $\bar{u}(t)=\left[\bar{u}_{1}, \bar{u}_{2}, \ldots, \bar{u}_{M}\right]^{T}$. Then, the system model $(1.1)$ is rewritten as

$$
\dot{x}(t)=A x(t)+B \Lambda v(t)+B \Lambda \sigma(\bar{u}(t)-v(t))+B \Theta^{*} \phi(x) .
$$

Next, we design an adaptive control signal $v(t)$ for 4.31 for desired system stabilization and state tracking. Notices that what if no actuator failure occurs in the plant, $u(t)=v(t)$ and the system model (4.31) is the controlled plant 11.1): $\dot{x}(t)=A x(t)+B \Lambda u(t)+B \Theta^{*} \phi(x)$. For this case, we can introduce the adaptive controller structure as

$$
v(t)=K_{1}^{T}(t) x(t)+K_{2}(t) r(t)+K_{3}(t) \phi(x)+K_{4}(t),
$$

where $K_{1}^{*} \in R^{n \times M}, K_{2} \in R^{M \times M}, K_{3}^{*} \in R^{M \times s}$ and $K_{4}^{*} \in R^{M}$ are the nominal versions of $K_{1}(t), K_{2}(t), K_{3}(t)$ and $K_{4}(t)$, with $K_{1}^{*}$ and $K_{2}^{*}$ designed for plant-model matching, and $K_{3}^{*}$ being chosen for compensation of the actuator nonlinearity, and $K_{4}^{*}(t)$ for compensation of the actuation error $B \Lambda(\bar{u}-v)$. To ensure desired compensation of uncertain actuator failures, some key system conditions are necessary which are derived as

$$
\begin{aligned}
& \operatorname{rank}\left(B_{a}\right)=\operatorname{rank}\left(B_{a} \mid A_{m}-A\right), \operatorname{rank}\left(B_{a}\right)=\operatorname{rank}\left(B_{a} \mid B_{m}\right), \\
& \operatorname{rank}\left(B_{a}\right)=\operatorname{rank}\left(B_{a} \mid B \Theta^{*}\right), \operatorname{rank}\left(B_{a}\right)=\operatorname{rank}(B),
\end{aligned}
$$

where $B_{a} \in R^{n \times(M-p)}$ is constructed by collecting the $M-p$ columns of $B, b_{j}, j \neq j_{1}, \cdots, j_{p}$ (assuming there are $p$ failed actuators at time $t$, that is, $u_{j}(t)=\bar{u}_{j}, j=j_{1}, \cdots, j_{p}$ ). Due to space limits, derivations for system conditions and control designs are not given here, and control designs are similar to the failure-free case.

For this case, we also obtain the results of closed-loop system signal boundedness and asymptotic tracking: $\lim _{t \rightarrow \infty} e(t)=0$, when $\Lambda$ in $(1.1)$ is an unknown diagonal or triangular matrix.

\section{Simulation Study}

To demonstrate the effectiveness of the proposed adaptive controller, a delta wing aircraft whose roll dynamics can be regulated by ailerons is derived from [8] as

$$
\begin{aligned}
& \dot{\phi}=p, \\
& \dot{p}=\theta_{1} \phi+\theta_{2} p+\left(\theta_{3}|\phi|+\theta_{4}|p|\right) p+\theta_{5} \phi^{3}+\theta_{6} \delta_{a},
\end{aligned}
$$

where $\phi$ and $p$ are the aircraft roll angle and roll rate, respectively, and $\delta_{a}$ is the differential aileron. The unknown system parameters are defined as $\theta_{1}=-0.018, \theta_{2}=0.015, \theta_{3}=-0.062, \theta_{4}=0.009, \theta_{5}=$ $-0.021, \theta_{6}=0.75$. Rewriting the model (5.35) in the form of 2.2) gives

$$
\left[\begin{array}{c}
\dot{\phi} \\
\dot{p}
\end{array}\right]=\left[\begin{array}{cc}
0 & 1 \\
\theta_{1} & \theta_{2}
\end{array}\right]\left[\begin{array}{l}
\phi \\
p
\end{array}\right]+\left[\begin{array}{l}
0 \\
1
\end{array}\right] \theta_{6}\left(u+\Theta^{*} \phi(x)\right)
$$

where $\Lambda=\theta_{6}$ is the system control effectiveness, $\Theta^{*}=\frac{1}{\theta_{6}}\left[\theta_{3}, \theta_{4}, \theta_{5}\right] \in R^{1 \times 3}, \phi(x)=\left[|\phi| p,|p| p, \phi^{3}\right]^{T} \in R^{3 \times 1}$. For simulations, the reference roll dynamics are defined by the second order transfer function:

$$
\frac{\phi_{r e f}}{\phi_{c m d}}=\frac{\omega_{n}^{2}}{s^{2}+2 \xi \omega_{n} s+\omega_{n}^{2}}
$$

\section{CDSR 118-6}


The above Laplace transform represents the desired command-to-response roll angle behavior, where $\phi_{\text {ref }}$ denotes the reference roll angle and $\phi_{c m d}$ is the commanded roll angle. $\left(\omega_{n}, \xi\right)$ are desired natural frequency and the damping ratio, respectively. Clearly, the matching conditions 2.3 can be given in the form below

$$
\begin{aligned}
{\left[\begin{array}{cc}
0 & 1 \\
\theta_{1} & \theta_{2}
\end{array}\right]+} & {\left[\begin{array}{l}
0 \\
1
\end{array}\right] \theta_{6} K_{10}^{T}=\left[\begin{array}{cc}
0 & 1 \\
-\omega_{n}^{2} & -2 \xi \omega_{n}
\end{array}\right], } \\
& {\left[\begin{array}{l}
0 \\
1
\end{array}\right] \theta_{6} K_{20}=\left[\begin{array}{c}
0 \\
\omega_{n}^{2}
\end{array}\right] . }
\end{aligned}
$$

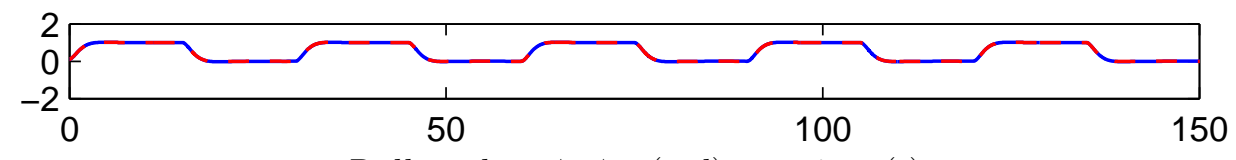

Roll angles: $\phi, \phi_{r e f}(\mathrm{rad})$, vs. time (s)

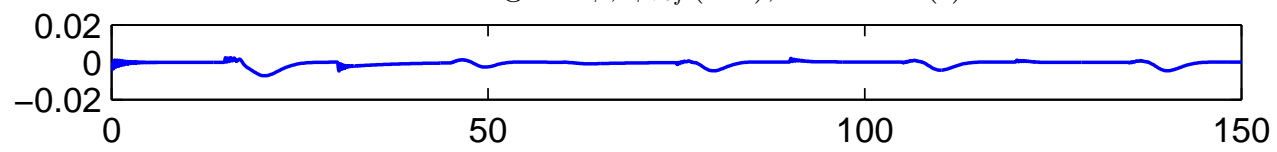

Tracking error $e_{1}=\phi-\phi_{\text {ref }}(\mathrm{rad})$ vs. time (s)

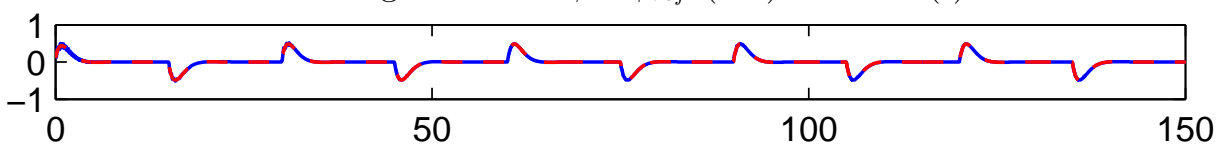

Roll rates: $p, p_{\text {ref }}(\mathrm{rad} / \mathrm{s})$, vs. time $(\mathrm{s})$

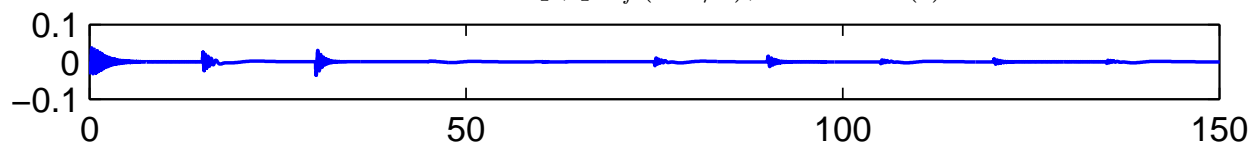

Tracking error $e_{2}=p-p_{\text {ref }}(\mathrm{rad} / \mathrm{s})$ vs. time $(\mathrm{s})$

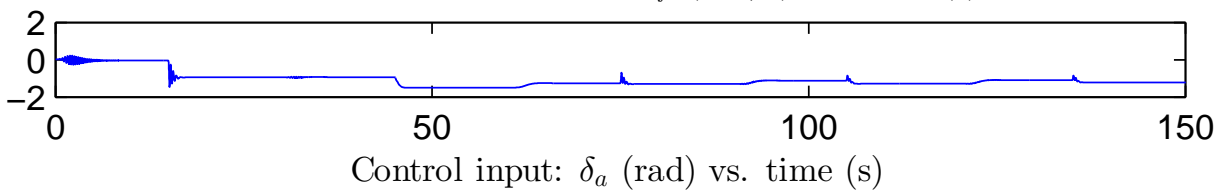

Fig. 1: System responses for the square wave $r(t)$ in 5.40 .

The reference system parameters are selected as: $\omega_{n}=1(\mathrm{rad} / \mathrm{s}), \xi=0.7$, and the reference input $r(t)$ is selected as a piecewise square signal shown below

$$
r(t)= \begin{cases}1 & 2 k T \leq t \leq(2 k+1) T, k=0,1,2, \ldots \\ 0 & \text { otherwise }\end{cases}
$$

Hence, the ideal gains are $K_{10}^{T}=-(1.3093,-1.8867)^{T}$ and $K_{20}=1.3333$, with adaption gain $\Gamma=100$. The constant matrix $Q \in R^{n \times n}$ is chosen as $Q=\operatorname{diag}\{1,10\}$ and thus $P$ which satisfies $P A_{m}+A_{m}^{T} P=-Q$ is

$$
P=\left[\begin{array}{ll}
4.5179 & 0.3767 \\
0.3767 & 2.8726
\end{array}\right]
$$

CDSR 118-7 
Applying adaptive law 2.12, we obtain simulation results showed in Fig. 1 for the system output $y(t)=$ $[\rho, p]^{T}$, reference output $y_{m}(t)=\left[\rho_{\text {ref }}, p_{r e f}\right]^{T}$, the tracking error $e(t)=\left[e_{1}, e_{2}\right]^{T}$, and control input signal $\delta_{a}$. From Figure 1 , it is obvious that our designed adaptive control signal $\delta_{a}$ as shown in Figure 1 can ensure the boundedness of all closed-loop system signals and the desired state tracking property.

\section{Conclusions}

This paper has studied adaptive control for an $M$-input linear time-invariant system with actuator failures and nonlinearities in presence of an unknown triangular matrix $\Lambda$. A simple adaptive controller has been designed without the use of an additional compensator system, which can ensure desired stability and state tracking properties. An extension of the developed adaptive control scheme has been extended to the case when $\Lambda$ is an unknown triangular matrix. Further results have been established for adaptive control of systems of the form (1.1), to deal with uncertainties of the actuation matrix $\Lambda$ and the failures of the actuators $u(t)$, to ensure desired system stability and state tracking properties.

\section{Acknowledgments}

The first author Dr. Liyan Wen was supported by the National Natural Science Foundations of China (grant 61703205 and 61803226) and the Youth Fund of Natural Science Fund of Jiangsu Province (grant

BK20170814), and she was currently a visiting assistant professor at Department of Electrical and Computer Engineering in University of Virginia.

\section{References}

[1] K. S. Narendra and A. M. Annaswamy, Stable Adaptive Systems, NJ: Prentice-Hall, Englewood Cliffs, 1989.

[2] P. A. Ioannou and J. Sun, Robust Adaptive Control, NJ: Prentice-Hall, Englewood Cliffs, 1996.

[3] G. Tao, Adaptive Control Design and Analysis, NJ: John Wiley and Sons, 2003.

[4] G. Tao, "Multivarible adaptive control: a survey," Automatica, vol. 50, pp. 2737-2764, 2014.

[5] G. Tao, S. H. Chen, X. D. Tang and S. M. Joshi, Adaptive Control of Systems with Actuator Failures, London: Springer, 2004.

[6] G. Tao, Adaptive Control Design and Analysis. Supplemental Notes [Online]. Available: http://www. people.virginia.edu/gt9s/wiley-book-notes.pdf, 2017.

[7] Z. Dydek, A. Annaswamy and E. Lavretsky. "Adaptive control and the NASA X-15-3 flight revisited," IEEE Control Systems Magazine, vol. 30, no. 3, pp. 32-48, 2010.

[8] E. Lavretsky and K. Wise, Robust Adaptive Control with Aerospace Applications, NY: Spring-Verlag, 2013.

[9] M. Malisoff, B. Sarsilmaz, T. Yucelen and J. Muse, "Tracking, parameter identification, and convergence rates for model reference adaptive control," in proceding of the American Control Conference, Milwaukee, WI, 27-29 June 2018, pp. 6810-6814. 